\title{
SHOCK REFLECTION FOR THE DAMPED P-SYSTEM
}

\author{
$\mathrm{BY}$ \\ LING HSIAO (Academy of Mathematics and System Sciences, Chinese Academy of Sciences, \\ Beijing, P. R. China) \\ AND
}

HAILIANG LI (Institute of Mathematics, Academia Sinica, Beijing, P. R. China and SISSA, Via Beirut, Trieste, Italy)

\begin{abstract}
The global existence and the asymptotic behavior of the weak entropy solution, the piecewise smooth solution with one shock discontinuity, on a strip domain is investigated in the present paper. We show that, for small smooth initial data and boundary value with only one small jump at $(x, t)=(0,0)$, the piecewise smooth solution with one shock discontinuity exists globally in time. The shock discontinuity begins from $(x, t)=(0,0)$, moves forward and reflects in a finite time at the boundary $x=1$ to form a 1-shock, which goes backward and reflects at $x=0$ also in a finite time to create a new 2 -shock. The shock strength decays exponentially and never disappears in finite time. As $t \rightarrow \infty$, this solution converges to a constant state determined by the initial and the boundary conditions.
\end{abstract}

1. Introduction. Consider the following $P$-system with damping in Lagrangian coordinates

$$
\begin{aligned}
& v_{t}-u_{x}=0, \\
& u_{t}+p_{x}=-\alpha u, \quad \alpha>0,
\end{aligned}
$$

where $v>0$ is the specific volume, $u$ is the velocity, and $p=p(v)$ is the pressure with $p^{\prime}(v)<0$. For simplicity, we consider in the present paper the typical case that $p(v)=v^{-\gamma}$ with $\gamma \geq 1$.

The system (1.1) can be viewed as the Euler equations with friction term added to the momentum equation, and can be used to describe the compressible flow through porous media.

\footnotetext{
Received September 21, 1999.

2000 Mathematics Subject Classification. Primary 35L45, 35L55, 35L65.

Key words and phrases. Damped $P$-system, shock reflection, asymptotic behavior, decay rates.

The first author was supported by the Special Funds of State Major Basic Research Projects (Grant No. 1999075107) and the Innovation Funds of AMSS, CAS of China.
} 
By approximating $(1.1)_{2}$ with Darcy's law, we obtain the following systems:

$$
\begin{gathered}
\tilde{v}_{t}=-\frac{1}{\alpha} p(\tilde{v})_{x x}, \\
\tilde{u}=-\frac{1}{\alpha} p(\tilde{v})_{x} .
\end{gathered}
$$

It has been shown in [5] that the smooth solutions of the Cauchy problem for (1.1) with initial data

$$
(v, u)(x, 0)=\left(v_{0}, u_{0}\right)(x), \quad v_{0}( \pm \infty)=\tilde{v}_{ \pm},
$$

tend time-asymptotically to the solutions of (1.2) with initial data

$$
\tilde{v}(x, 0)=v^{*}(x+b)
$$

where $v^{*}$ is the similarity solution to $(1.2)_{1}$ with $v^{*}(\eta)=\tilde{v}_{ \pm}$as $\eta \rightarrow \pm \infty\left(\eta=\frac{x}{\sqrt{1+t}}\right)$, and $b$ is a constant. Namely, the nonlinear diffusive phenomena of smooth solutions to (1.1) occurs due to the damping mechanism. Based on the resolution of the perturbed Riemann problem for $(1.1)$ in $[13,14]$, the nonlinear diffusive phenomena of entropy weak solutions to (1.1) was shown in [8]. There are other related results to [5], such as $[3,4,6,7,11,12,23,27]$ for smooth solutions, $[2,18,19,21,25,26]$ for weak solutions, and the references therein.

Recently, the initial boundary value problems for the hyperbolic system (1.1) and the comparison of its time-asymptotic behavior to the corresponding initial boundary value problems for the reduced decoupled system (1.2) were discussed in $[20,24]$ on a quarter plane in $(x, t)$ and in $[9,16]$ on a strip domain with different boundary conditions, respectively. All of these results concern smooth solutions. In the present paper, we investigate the global existence and the qualitative behavior of weak entropy solutions to the initial boundary value problems (IBVP) for $(1.1)$ on $\Omega \equiv[0,1] \times[0,+\infty)$ with initial data

$$
(v, u)(x, 0)=\left(v_{0}, u_{0}\right)(x), \quad x \in(0,1)
$$

and boundary values

$$
u(0, t)=u_{1}(t), \quad u(1, t)=u_{2}(t), \quad t \geq 0,
$$

where $\left(v_{0}, u_{0}\right)(x)$ is a given smooth function with $v_{0}(x)>0$ and the function $u_{i}(t)$ $(i=1,2)$ is smooth with

$$
u_{i}(t) \rightarrow 0 \text { as } t \rightarrow \infty, i=1,2,
$$

but there are discontinuities initially at the end points $x=0$ or $x=1$.

We are interested in the questions whether the global weak entropy solutions to IBVP (1.1), (1.3), and (1.4) exist and whether they have the same large-time behavior compared to those of the IBVP (1.2) with initial data

$$
\tilde{v}(x, 0)=\tilde{v}_{0}(x), \quad x \in[0,1]
$$

and the corresponding boundary values

$$
p_{x}(0, t)=f_{1}(t), \quad p_{x}(1, t)=f_{2}(t), \quad t \geq 0
$$

where $\tilde{v}_{0}(x)$ is a given function with $\tilde{v}_{0}(x)>0$ and $f_{i}(t)=-\left(a u_{i}(t)+\dot{u}_{i}(t)\right), i=1,2$. 
For simplicity, we first consider in the present paper the problem of shock reflection, which is important in the theory of fluid dynamics (see [1] for details).

Define

$$
\begin{aligned}
& Q(t)=\int_{0}^{1} v_{0}(x) d x+\int_{0}^{t}\left(u_{2}-u_{1}\right)(\tau) d \tau \\
& \widetilde{Q}(t)=\int_{0}^{1} \tilde{v}_{0}(x) d x+\int_{0}^{t}\left(u_{2}-u_{1}\right)(\tau) d \tau+\left(u_{2}-u_{1}\right)(t)-\left(u_{2}-u_{1}\right)(0),
\end{aligned}
$$

and assume that there are positive constants $Q_{1}, Q_{2}, \widetilde{Q}_{1}$, and $\widetilde{Q}_{2}$ such that

$$
\begin{array}{ll}
0<Q_{1} \leq Q(t) \leq Q_{2}<+\infty, & t \geq 0, \\
0<\widetilde{Q}_{1} \leq \widetilde{Q}(t) \leq \widetilde{Q}_{2}<+\infty, \quad t \geq 0 .
\end{array}
$$

Let $\bar{v}_{1}=Q(+\infty)$ and $\bar{v}_{2}=\widetilde{Q}(+\infty)$. Then it follows that $\bar{v}_{1}=\bar{v}_{2}$, provided that

$$
\int_{0}^{1} \tilde{v}_{0}(x) d x-\left(u_{2}-u_{1}\right)(0)=\int_{0}^{1} v_{0} d x .
$$

Denote

$$
g(t)=\int_{t}^{+\infty}\left(u_{2}-u_{1}\right)(\tau) d \tau
$$

which is bounded due to (1.8) and (1.10).

Suppose that there is only one jump of velocity at the point $(x, t)=(0,0)$ initially. The main results in this case show that the global weak solution, which is a piecewise smooth solution with one shock discontinuity at any fixed $t>0$, to the IBVP (1.1), (1.3), and (1.4) exists provided that $\left(v_{0}, u_{0}\right) \in C^{3}((0,1))$ with $\left|v_{0}-\bar{v}_{1}\right|_{C^{2}}+\left|u_{0 x}\right|_{C^{1}} \ll 1$, $\left(u_{1}, u_{2}\right) \in C^{3} \cap H^{3}((0,+\infty))$ with $\left|\left(u_{1}, u_{2}\right)\right|_{C^{3}}+\left|\left(u_{1}, u_{2}\right)\right|_{H^{3}}+|g|_{C^{0}} \ll 1$, and the initial jump is sufficiently small. The shock discontinuity begins from $(x, t)=(0,0)$, moves forward and reflects in a finite time at the boundary $x=1$ to form a 1-shock, which goes backward and reflects at $x=0$ also in a finite time to create a new 2 -shock. The shock strength decays exponentially and never disappears in any finite time. In addition, the weak entropy solution has the same large-time behavior compared to that for the IBVP (1.2), (1.6), and (1.7) in the sense that they have the same asymptotic state and the same convergence rate, provided that (1.12) holds. Similar results are true for the case that there are initially two jumps of velocity at the points $(x, t)=(0,0)$ and $(x, t)=(1,0)$, respectively. However, it is much more complicated to deal with, since the two shock discontinuities coming from $(x, t)=(0,0)$ and $(x, t)=(1,0)$ originally will interact in finite time to form two new shock discontinuities, which move and reflect at boundaries to form shock discontinuities in the other family.

For the Cauchy problem when the initial data is a perturbed Riemann data, the global existence of a piecewise solution for (1.1) was solved with the help of the characteristic method in $[13,14]$. Away from the shock discontinuity, the uniform bounds of specific volume can be controlled in terms of those of the Riemann invariants $s$ and $r$ and their derivatives $s_{x}$ and $r_{x}$, where the condition $1 \leq \gamma<3$ was required to obtain the bounds of $s_{x}$ and $r_{x}$. However, this method cannot be extended directly to our case since the successive reflection of the shock discontinuity at the boundaries causes the jump in the 
specific volume and such a process will never stop in any finite time. In addition, due to the boundary effects, the value of $v(x, t)$ may not be controlled by the bounds of $v_{0}(x)$. Instead, the energy method is used in the present paper to overcome difficulties. On the existence domain, under the a priori assumptions that the shock strength is sufficiently weak and that away from the discontinuity it holds that $0<v_{m} \leq v(x, t) \leq v_{M}$ and $\left|v_{M I}-v_{m}\right|+\left|v_{x}\right|+\left|u_{x}\right| \ll 1$, where $v_{m}$ and $v_{M}$ are two constants satisfying (2.18), we construct the shock discontinuity, obtain the exponential decay of its strength locally, control the bounds of $r_{x x}, s_{x x}, r_{x x x}$, and $s_{x x x}$ away from the discontinuity, and gain the exponential decay of the jump of $r_{x}, s_{x}, r_{x x}$, and $s_{x x}$ on both sides of the discontinuity. Then, based on the observation that each time after reflection at the boundary, the increase of shock strength, for weak shock waves, will be cancelled by the dissipative effect of frictional damping, we can estimate, via energy methods, the uniform bounds of $v(x, t)$ and $\left|v_{x}\right|+\left|u_{x}\right|$ in terms of those of the initial data and boundary values for any $t>0$. Moreover, we can verify how the boundary effects influence the time-convergence rates of the piecewise smooth solution. The methods and approaches used in the present paper are applied for $\gamma \geq 1$. Also, they can be extended to solve the problem for general pressure $p(v)$ satisfying $p^{\prime}(v)<0<p^{\prime \prime}(v)$.

This paper is arranged as follows. In Sec. 2, we investigate the case of one shock reflection. The main result for this case is given in Sec. 2.1. The necessary estimates of the piecewise smooth solution will be obtained locally in Secs. 2.2-2.3. The global existence of it and its time decay rate are obtained in Secs. 2.4-2.5, respectively. The result on two-shock reflection is given in Sec. 3 .

Notation. From now on, $L^{2}$ will denote the usual space of square integrable functions with norm $\|\cdot\|$ on $[0,1]$ or $R_{+}=(0,+\infty)$, and $H^{l}(l \geq 0)$ the usual Sobolev space with norm $\|\cdot\|_{l}$ on $[0,1]$ or $R_{+}$. We assume that for any of the above norms written as $\|\cdot\|_{n}$, the norm of a vector-valued function $\left(g_{1}, g_{2}, g_{3}\right)$ is given by

$$
\left\|\left(g_{1}, g_{2}, g_{3}\right)\right\|_{n}=\sum_{i=1}^{3}\left\|g_{i}\right\|_{n} .
$$

\section{One-shock reflection.}

2.1. Main results. The characteristic speeds of system (1.1) are $\lambda=-\sqrt{\gamma} v^{-(\gamma+1) / 2}$ for the 1 -family and $\mu=\sqrt{\gamma} v^{-(\gamma+1) / 2}$ for the 2-family. For simplicity, we assume that $\alpha=1$.

An $i$-shock discontinuity, $i=1,2$, in a solution of (1.1) is characterized by the RankineHugoniot condition and the Lax entropy condition, i.e., along the discontinuity $x=x_{i}(t)$ it follows that

$$
\left\{\begin{array}{l}
\dot{x}_{1}(t)=-\sqrt{-[p(v)]_{1} /[v]_{1}}, \\
{[u]_{1}=\sqrt{-[p(v)]_{1} /[v]_{1}} \cdot[v]_{1},} \\
\lambda\left(v\left(x_{1}(t)-0, t\right)\right)>\dot{x}_{1}(t)>\lambda\left(v\left(x_{1}(t)+0, t\right)\right),
\end{array}\right.
$$


or

$$
\left\{\begin{array}{l}
\dot{x}_{2}(t)=\sqrt{-[p(v)]_{2} /[v]_{2}}, \\
{[u]_{2}=-\sqrt{-[p(v)]_{2} /[v]_{2}} \cdot[v]_{2},} \\
\mu\left(v\left(x_{2}(t)-0, t\right)\right)>\dot{x}_{2}(t)>\mu\left(v\left(x_{2}(t)+0, t\right)\right),
\end{array}\right.
$$

where and from now on

$$
[F]_{i}=F\left(x_{i}(t)+0, t\right)-F\left(x_{i}(t)-0, t\right), \quad i=1,2 .
$$

Let $u_{-}=u_{1}(0)$ and $u_{+}=\lim _{x \rightarrow 0+} u_{0}(x)$. In this section, we consider the IBVP (1.1), (1.3), and (1.4) in the case

$$
u_{-}>u_{+}, \quad u_{2}(0)=\lim _{x \rightarrow 1-0} u_{0}(x) .
$$

Let $v_{+}=\lim _{x \rightarrow 0+} v_{0}(x)$ and $v_{-}$be the solution to the following equation:

$$
\left(u_{-}-u_{+}\right)^{2}=\left(p\left(v_{-}\right)-p\left(v_{+}\right)\right)\left(v_{+}-v_{-}\right),
$$

with $v_{-}<v_{+}$.

Denote

$$
\begin{aligned}
\delta_{0} & =\left|v_{+}-v_{-}\right|+\left|u_{+}-u_{-}\right|, \\
\mu_{0} & =\max _{t \geq 0}\left\{\sum_{i=0}^{3}\left(\left|\frac{d^{i} u_{1}(t)}{d t^{i}}\right|+\left|\frac{d^{i} u_{2}(t)}{d t^{i}}\right|\right)+|g(t)|+\left\|\left(u_{1}, u_{2}\right)\right\|_{3}\right\}, \\
N_{1} & =\max _{x \in(0,1)} \sum_{i=0}^{2}\left|\partial_{x}^{i}\left(v_{0}(x)-\bar{v}_{1}\right)\right|+\max _{x \in(0,1)} \sum_{i=1}^{2}\left|\partial_{x}^{i} u_{0}(x)\right|, \\
N_{2} & =\max _{x \in(0,1)}\left|\partial_{x}^{3}\left(v_{0}(x)-\bar{v}_{1}\right)\right|+\max _{x \in(0,1)}\left|\partial_{x}^{3} u_{0}(x)\right|, \\
A(t) & =\sqrt{\int_{t}^{t+1}\left(\sum_{i=0}^{3}\left(\left|\frac{d^{i} u_{1}(t)}{d t^{i}}\right|^{2}+\left|\frac{d^{i} u_{2}(t)}{d t^{i}}\right|^{2}\right)+g^{2}\right)(\tau) d \tau .}
\end{aligned}
$$

We have the following main result.

Theorem 2.1. Assume that $\delta_{0} \ll 1,\left(u_{0}, v_{0}\right) \in C^{3}((0,1))$ with $N_{1}+N_{2}<+\infty$, and $u_{i} \in C^{3} \cap H^{3}((0,+\infty))(i=1,2)$. Then there exists an $\varepsilon_{0}>0$ such that if $N_{1}+\mu_{0}<\varepsilon_{0}$, the global weak entropy solution $(u, v)(x, t)$ to the IBVP $(1.1),(1.3),(1.4)$, and (2.3) exists, namely, a continuous and piecewise smooth curve $x=x(t): R_{+} \rightarrow[0,1]$, satisfying $\dot{x}(t) \neq 0, \dot{x}(t)=\dot{x}_{1}(t)$ for $\dot{x}(t)<0$ and $\dot{x}(t)=\dot{x}_{2}(t)$ for $\dot{x}(t)>0$, exists such that $(u, v)(x, t) \in C^{3}$ for $x \neq x(t)$ and along $x=x(t)$, the Rankine-Hugoniot condition and the Lax entropy condition hold. In addition, as $t$ tends to infinity,

$$
\left|\partial_{x}^{i}((u, v)(x(t)+0, t)-(u, v)(x(t)-0, t))\right| \sim O(1) e^{-\beta_{1} t} \rightarrow 0, \quad i=0,1,2,
$$

and

$$
\left\|\left(u, v-\bar{v}_{1}\right)(\cdot, t)\right\|_{2}^{2} \sim O(1)\left(e^{-\beta_{2} t}+A(t)\right) \rightarrow 0,
$$

with two positive constants $\beta_{1}$ and $\beta_{2}$. 
REMARK 2.2. 1) The similar result is true for general pressure $p(v)$ with $p^{\prime}(v)<0<$ $p^{\prime \prime}(v)$.

2) The method used here can also be used to obtain the global existence of a piecewise smooth solution with shock discontinuity or rarefaction wave for the piston problem for (1.1) on a quarter plane, and for the perturbed Riemann problem for (1.1).

To compare the time-asymptotic behavior of the weak entropy solutions of the IBVP (1.1), (1.3), (1.4), and (2.3) to the solutions of the IBVP (1.1), (1.6), and (1.7), we first give the global existence result for the IBVP (1.1), (1.6), and (1.7).

Assume that $f_{1}(0)=\lim _{x \rightarrow 0+} p\left(\tilde{v}_{0}(x)\right)_{x}$ and $f_{2}(0)=\lim _{x \rightarrow 1-0} p\left(\tilde{v}_{0}(x)\right)_{x}$, and the corresponding compatibility conditions for higher-order derivatives also hold. Then we have

Lemma $2.3([16])$. Assume that $\tilde{v}_{0}(x) \in H^{3}, u_{i} \in H^{3}(i=1,2)$. Then, there is an $\varepsilon_{1}>0$ such that if $\left\|\left(\tilde{v}_{0}-\bar{v}_{2}\right)\right\|_{3}+\mu_{0} \leq \varepsilon_{1}$, a global solution $(\tilde{v}, \tilde{u})$ to (1.2), (1.6), and (1.7) exists and satisfies

$$
\left(\left\|\left(\tilde{v}-\bar{v}_{2}\right)(\cdot, t)\right\|_{3}^{2}+\|u(\cdot, t)\|_{2}^{2}\right) \sim C\left(e^{-\beta_{3} t}+A(t)\right) \rightarrow 0, \quad \text { as } t \rightarrow+\infty,
$$

with $\beta_{3}$ a positive constant.

With the help of Theorem 2.1 and Lemma 2.3, we can compare the time-asymptotic behavior of the solutions.

TheOREm 2.4. Let $(v, u)$ and $(\tilde{v}, \tilde{u})$ be the solutions to the IBVP $(1.1),(1.3),(1.4)$, and (2.3), and the IBVP (1.2), (1.6), and (1.7), respectively. Assume that (1.12) holds. Then, there is an $\varepsilon_{2}>0$ such that if $N_{1}+\left\|\left(\tilde{v}_{0}-\bar{v}_{2}\right)\right\|_{3}+\mu_{0} \leq \varepsilon_{2}$, it follows that

$$
\|(v-\tilde{v}, u-\tilde{u})(\cdot, t)\|_{2}^{2} \sim C\left(e^{-\beta_{4} t}+A(t)\right) \rightarrow 0, \quad \text { as } t \rightarrow+\infty,
$$

with $\beta_{4}$ a positive constant.

2.2. Qualitative behavior of the weak entropy solution. Now, we construct the local discontinuous solution for the IBVP (1.1), (1.3), (1.4), and (2.3), and investigate the corresponding qualitative behavior of the weak entropy solution.

Introduce Riemann invariants

$$
\begin{aligned}
& s=u+\phi(v), \\
& r=u-\phi(v),
\end{aligned}
$$

where $\phi$ is defined by

$$
\phi(v)=\left\{\begin{array}{l}
\frac{2 \sqrt{\gamma}}{\gamma-1} v^{-(\gamma-1) / 2}, \quad \gamma \neq 1 \\
-\ln v, \quad \gamma=1
\end{array}\right.
$$

Define

$$
\begin{aligned}
& D_{-}=\partial_{t}+\lambda \partial_{x}, \\
& D_{+}=\partial_{t}+\mu \partial_{x} .
\end{aligned}
$$


Then the system (1.1) can be written as

$$
\begin{aligned}
& D_{-} r=-\frac{1}{2}(r+s), \\
& D_{+} s=-\frac{1}{2}(r+s),
\end{aligned}
$$

and the corresponding initial and boundary values are

$$
\begin{aligned}
(r, s)(x, 0) & =\left(r_{0}, s_{0}\right)(x)=:\left(u_{0}-\phi\left(v_{0}\right), u_{0}+\phi\left(v_{0}\right)\right)(x), \quad x \in(0,1), \\
s(0, t) & =-r(0, t)+2 u_{1}(t), \quad t \geq 0, \\
r(1, t) & =-s(1, t)+2 u_{2}(t), \quad t \geq 0 .
\end{aligned}
$$

First of all, by using the initial data on $0<x<1$ and the boundary condition on $x=1$, we are able to get a unique $C^{1}$ solution $(\tilde{r}, \tilde{s})$ on the domain

$G_{+}=\left\{(x, t) \mid x_{+}(t) \leq x \leq 1,0 \leq t \leq \widehat{T}_{+}, \dot{x}_{+}(t)=\mu\left(v\left(x_{+}(t), t\right)\right), x_{+}(0)=0, x_{+}\left(\widehat{T}_{+}\right) \leq 1\right\}$

with the specific volume bounded and $\left|\left(\tilde{r}_{x}, \tilde{s}_{x}\right)\right|_{C^{0}} \ll 1$ provided that $v_{0}(x)>0$ and $\left|\left(v_{0}, u_{0}\right)_{x}\right|_{C^{0}}+\left|u_{2 t}\right|_{C^{0}} \ll 1$.

By the argument used by $\mathrm{Li}$ and $\mathrm{Yu}$ in [17] to establish the local existence theorem, the discontinuous initial-boundary value problem (1.1), (1.3), (1.4), and (2.3) admits a unique discontinuous solution $(r, s)$ on a local domain

$$
\Omega(\widehat{T})=:\left\{(x, t) \mid 0 \leq x \leq 1,0 \leq t \leq \widehat{T} \leq \widehat{T}_{+}\right\}
$$

in the class of piecewise continuous and piecewise smooth functions. This solution contains only a forward shock $x=x_{2}(t)$ passing through $(0,0)$. It is known, due to the entropy condition proposed in the initial-boundary data, that $x=x_{2}(t)$ must be located on the right side of $x=x_{+}(t)$, and therefore the solution on the right side of $x=x_{2}(t)$ will be furnished by $(\tilde{r}, \tilde{s})$. Moreover, it can be shown that the specific volume is bounded, $\left|\left(r_{x}, s_{x}\right)\right| \ll 1$ for $x \neq x_{2}(t)$, and $\left|[v]_{2}\right| \ll 1$, provided that $v_{0}(x)>0$ and $\left|\left(v_{0}, u_{0}\right)_{x}\right|_{C^{0}}+\left|\left(u_{1 t}, u_{2 t}\right)\right|_{C^{0}}+\delta_{0} \ll 1$.

For any $T_{2}>T_{1} \geq 0$ denote

$$
\begin{array}{ll}
\Omega_{i}^{-}\left(T_{1}, T_{2}\right)=\left\{(x, t) \mid 0<x<x_{i}(t), T_{1} \leq t<T_{2}\right\}, & i=1,2, \\
\Omega_{i}^{+}\left(T_{1}, T_{2}\right)=\left\{(x, t) \mid x_{i}(t)<x<1, T_{1} \leq t<T_{2}\right\}, & i=1,2 .
\end{array}
$$

Now, without loss of generality, we assume that the piecewise smooth solution exists on $\Omega\left(T_{1}\right)$ with $T_{1} \geq \widehat{T}$ and $x_{2}\left(T_{1}\right)<1$, smooth on domain $\Omega_{s}\left(T_{1}\right)=: \Omega_{2}^{+}\left(0, T_{1}\right) \cup \Omega_{2}^{-}\left(0, T_{1}\right)$, and assume that on domain $\Omega_{s}\left(T_{1}\right)$ it holds that

$$
v_{m}<v(x, t)<v_{M}, \quad\left|v_{M}-v_{m}\right|+\left|u_{x}(x, t)\right|+\left|v_{x}(x, t)\right| \leq \eta \ll 1,
$$

with two constants $v_{m}$ and $v_{M}$ satisfying

$$
0<v_{m}<\min _{x \in[0,1]}\left\{\bar{v}_{1}, v_{0}(x)\right\} \leq \max _{x \in[0,1]}\left\{\bar{v}_{1}, v_{0}(x)\right\}<v_{M},
$$

and along $x=x_{2}(t)$ it holds that

$$
\left|[v]_{2}\right| \ll 1 .
$$

We next investigate the qualitative behaviors of the piecewise smooth solution, particularly those on both sides of the discontinuity, and the decay of the shock strength under the a priori assumptions (2.17) and (2.19). 
Define

$$
\begin{gathered}
K_{l}=\left(v_{2}^{+}\right)^{(\gamma+1) / 2}+(-1)^{l}\left(v_{2}^{-}\right)^{(\gamma+1) / 2}+(-1)^{l+1} \frac{1}{2} \sqrt{\gamma} \dot{x}_{2}[v]_{2}, \quad l=3,4, \\
A_{2}=\sqrt{\gamma}+\frac{1}{2 \sqrt{\gamma}}\left(\left(\mu_{2}^{-}\right)^{2}+\left(\dot{x}_{2}\right)^{2}\right) \frac{\mu_{2}^{-}}{\dot{x}_{2}}, \quad J_{2}^{ \pm}=\frac{\dot{x}_{2}}{\mu_{2}^{ \pm}}, \\
a_{s}^{+}=\left(1-J_{2}^{+}\right)\left(A_{2}-\mu_{2}^{+} K_{4}\right), \quad a_{s}^{-}=\left(1-J_{2}^{-}\right)\left(A_{2}-2 \mu_{2}^{-}\right), \\
a_{r}^{+}=\left(1+J_{2}^{+}\right)\left(A_{2}-\mu_{2}^{+} K_{3}\right), \quad a_{r}^{-}=A_{2}\left(1+J_{2}^{-}\right),
\end{gathered}
$$

where $\mu_{i}^{ \pm}=\mu\left(v\left(x_{i}(t) \pm 0, t\right)\right)$ and $F_{i}^{ \pm}=F\left(x_{i}(t) \pm 0, t\right), i=1,2$.

Differentiating $(2.2)_{2}$ with respect to $t$, and using $(2.12)$, we have, after a careful calculation, the following lemma.

LEMMA 2.5. Assume that the piecewise smooth solution of the IBVP (1.1), (1.3), (1.4), and (2.3) exists on $\Omega\left(T_{1}\right)$. Then, along $x=x_{2}(t)$, it holds that

$$
a_{r}^{-}\left(r_{2}^{-}\right)_{x}+a_{s}^{-}\left(s_{2}^{-}\right)_{x}=a_{s}^{+}\left(s_{2}^{+}\right)_{x}+a_{r}^{+}\left(r_{2}^{+}\right)_{x}+2 \sqrt{\gamma} J_{2}^{-}[v]_{2}
$$

and

$$
\begin{aligned}
-\left\{\frac{\left\{2 \sqrt{-\gamma[p(v)]_{2}[v]_{2}}+\left(\gamma\left(v_{2}^{-}\right)^{-(\gamma+1)}[v]_{2}-[p(v)]_{2}\right)\left(v_{2}^{-}\right)^{-(\gamma+1) / 2}\right\}}{[v]_{2} \sqrt{-[p(v)]_{2}[v]_{2}}} D_{t}^{+}[v]_{2}\right. \\
=2\left(v_{2}^{-}\right)^{(\gamma+1) / 2} \sqrt{-[p(v)]_{2} /[v]_{2}} \\
\quad+\frac{2\left(\sqrt{\gamma}\left(v_{2}^{-}\right)^{-(\gamma+1) / 2}-\sqrt{-[p(v)]_{2} /[v]_{2}}\right)}{[v]_{2}}\left(s_{2}^{-}\right)_{x} \\
\quad-\frac{\left(\sqrt{\gamma}\left(v_{2}^{+}\right)^{-(\gamma+1) / 2}+\sqrt{-[p(v)]_{2} /[v]_{2}}\right) K_{3}}{[v]_{2}}\left(r_{2}^{+}\right)_{x} \\
\quad+\frac{\left(-\sqrt{\gamma}\left(v_{2}^{+}\right)^{-(\gamma+1) / 2}+\sqrt{-[p(v)]_{2} /[v]_{2}}\right) K_{4}}{[v]_{2}}\left(s_{2}^{+}\right)_{x},
\end{aligned}
$$

where $D_{t}^{+}=: \partial_{t}+\dot{x}_{2}(t) \partial_{x}$.

Then, we have the following exponential decay for the shock strength along $x=x_{2}(t)$.

Lemma 2.6. Assume that the piecewise smooth solution of the IBVP (1.1), (1.3), (1.4), and (2.3) exists on $\Omega\left(T_{1}\right)$. Then, under the assumptions of Theorem 2.1, it follows that, along $x=x_{2}(t)$,

$$
\begin{gathered}
-\frac{1}{[v]_{2}} D_{t}^{+}\left([v]_{2}\right) \in\left[B_{1}, B_{2}\right], \quad t \in\left[0, T_{1}\right], \\
\delta_{0} e^{-B_{2} t} \leq v\left(x_{2}(t)+0, t\right)-v\left(x_{2}(t)-0, t\right) \leq \delta_{0} e^{-B_{1} t}, \quad t \in\left[0, T_{1}\right],
\end{gathered}
$$

with constants $B_{i}>0(i=1,2)$, provided that $(2.17)$ on $\Omega_{s}\left(T_{1}\right)$ and (2.19) hold.

Proof. By (2.17), it follows from (2.12) that for any $(x, t) \in \Omega_{s}\left(T_{1}\right)$,

$$
\left|s_{x}\right|+\left|r_{x}\right| \leq O(1) \eta \ll 1 .
$$

By the Lax entropy condition $(2.2)_{3}$ and (2.17), we have

$$
\mu_{M}=: \max _{v \in\left[v_{m}, v_{M}\right]} \mu(v)>\dot{x}_{2}(t)>\mu_{m}=: \min _{v \in\left[v_{m}, v_{M}\right]} \mu(v) .
$$


Since

$$
\begin{gathered}
\frac{\left\{2 \sqrt{-\gamma[p(v)]_{2}[v]_{2}}+\left(\gamma\left(v_{2}^{-}\right)^{-(\gamma+1)}[v]_{2}-[p(v)]_{2}\right)\left(v_{2}^{-}\right)^{-(\gamma+1) / 2}\right\}}{\sqrt{-[p(v)]_{2}[v]_{2}}} \\
=2 \sqrt{\gamma}\left\{1+\frac{\left(\left(\mu\left(v_{2}^{-}\right)\right)^{2}+\left(\dot{x}_{2}(t)\right)^{2}\right) \mu\left(v_{2}^{-}\right)}{2 \gamma \dot{x}_{2}(t)}\right\}
\end{gathered}
$$

it follows, in terms of $(2.17)$ and $(2.25)$, that

$$
\begin{aligned}
2 \sqrt{\gamma}\left(1+\frac{\mu_{m}^{3}}{\gamma \mu_{M}}\right) & <\frac{\left\{2 \sqrt{-\gamma[p(v)]_{2}[v]_{2}}+\left(\gamma\left(v_{2}^{-}\right)^{-(\gamma+1)}[v]_{2}-[p(v)]_{2}\right)\left(v_{2}^{-}\right)^{-(\gamma+1) / 2}\right\}}{\sqrt{-[p(v)]_{2}[v]_{2}}} \\
& <2 \sqrt{\gamma}\left(1+\frac{\mu_{M}^{3}}{\gamma \mu_{m}}\right) .
\end{aligned}
$$

By (2.17) and (2.19), we can verify that

$$
\sqrt{-\frac{[p(v)]_{2}}{[v]_{2}}} \sim\left(\mu\left(v_{2}^{-}\right)-O(1)[v]_{2}\right)
$$

or

$$
\sqrt{-\frac{[p(v)]_{2}}{[v]_{2}}} \sim\left(\mu\left(v_{2}^{+}\right)+O(1)[v]_{2}\right) .
$$

Therefore, we estimate the last three terms in the right-hand side of $(2.21)$, in terms of $(2.17),(2.19),(2.25),(2.27)$, and $(2.28)$, as follows:

$$
\begin{gathered}
\left|\frac{2\left(\sqrt{\gamma}\left(v_{2}^{-}\right)^{-(\gamma+1) / 2}-\sqrt{-[p(v)]_{2} /[v]_{2}}\right)}{[v]_{2}}\left(s_{2}^{-}\right)_{x}\right| \leq O(1) \eta, \\
\left|\frac{\left(\sqrt{\gamma}\left(v_{2}^{+}\right)^{-(\gamma+1) / 2}+\sqrt{-[p(v)]_{2} /[v]_{2}}\right) K_{3}}{[v]_{2}}\left(r_{2}^{+}\right)_{x}\right| \\
\leq 2 \mu_{M}\left|\left(\frac{1}{2} \dot{x}_{2}(t)-\frac{[\mu(v)]_{2}}{[v]_{2} \mu\left(v_{2}^{+}\right) \mu\left(v_{2}^{-}\right)}\right)\left(r_{2}^{+}\right)_{x}\right| \leq O(1) \eta,
\end{gathered}
$$

and

$$
\left|\frac{\left(-\sqrt{\gamma}\left(v_{2}^{+}\right)^{-(\gamma+1) / 2}+\sqrt{-[p(v)]_{2} /[v]_{2}}\right) K_{4}}{[v]_{2}}\left(s_{2}^{+}\right)_{x}\right| \leq O(1) \eta,
$$

where we have used (2.24).

Using (2.25), (2.26), and (2.29)-(2.31), we obtain (2.22) and (2.23) with

$$
B_{1}=\frac{\mu_{m}}{2 \mu_{M}} \cdot\left(1+\frac{\mu_{M}^{3}}{\gamma \mu_{m}}\right)^{-1}, \quad B_{2}=\frac{1}{2}\left(1+\frac{\mu_{m}^{3}}{\gamma \mu_{M}}\right)^{-1},
$$

provided that (2.17) and (2.19) hold. 
What we will do next is to obtain the bounds of $\left(r_{x x}, s_{x x}\right)(x, t)$ and $\left(r_{x x x}, s_{x x x}\right)(x, t)$ on $\Omega_{s}\left(T_{1}\right)$ and the decay rates of $\left[r_{x}\right]_{2},\left[s_{x}\right]_{2},\left[r_{x x}\right]_{2}$, and $\left[s_{x x}\right]_{2}$ under the a priori assumptions (2.17) and (2.19).

Define

$$
\begin{aligned}
& a(v)=\frac{1}{4}(\gamma+1) v^{(\gamma-3) / 4}, \quad c(v)=v^{-(\gamma+1) / 4}, \\
& h(v)=\left\{\begin{array}{l}
\frac{2}{3-\gamma} v^{-(\gamma-3) / 4}, \quad \gamma \neq 3, \\
\ln v^{1 / 2}, \quad \gamma=3,
\end{array}\right.
\end{aligned}
$$

and set

$$
\begin{array}{ll}
Y_{1}=c(v) s_{x}+h(v), & Z_{1}=c(v) r_{x}+h(v), \\
Y_{2}=\left(Y_{1}\right)_{x}, & Z_{2}=\left(Z_{1}\right)_{x} .
\end{array}
$$

It follows from $(2.13)$ that for $(x, t) \in \Omega_{s}\left(T_{1}\right)$,

$$
\begin{aligned}
& D_{+} Y_{1}=-a(v) Y_{1}^{2}-b_{1}(x, t) Y_{1}+f_{3}(x, t), \\
& D_{-} Z_{1}=-a(v) Z_{1}^{2}-b_{2}(x, t) Z_{1}+f_{4}(x, t),
\end{aligned}
$$

where

$$
\begin{aligned}
& b_{1}(x, t)=b_{2}(x, t)=\frac{1}{2}-2 a(v) h(v), \\
& f_{3}(x, t)=f_{4}(x, t)=h(v)\left(\frac{1}{2}-2 a(v) h(v)\right),
\end{aligned}
$$

and that

$$
\begin{aligned}
& D_{+} Y_{2}=-b_{3}(x, t) Y_{2}+f_{5}(x, t), \\
& D_{-} Z_{2}=-b_{4}(x, t) Z_{2}+f_{6}(x, t),
\end{aligned}
$$

where

$$
\begin{aligned}
b_{3}(x, t)= & \frac{1}{2}-\frac{1}{2}(\gamma+1) v^{-1} s_{x}+\mu^{\prime} v_{x}, \\
b_{4}(x, t)= & \frac{1}{2}-\frac{1}{2}(\gamma+1) v^{-1} r_{x}-\mu^{\prime} v_{x}, \\
f_{5}(x, t)= & -\mu^{\prime}(v) v_{x}\left(c(v) s_{x}+h(v)\right)-a^{\prime}(v) v_{x}\left(c(v) s_{x}\right)^{2} \\
& +\frac{1}{2} h^{\prime}(v)\left(1+(\gamma+1) v^{-1} s_{x}\right) v_{x}, \\
f_{6}(x, t)= & -\lambda^{\prime}(v) v_{x}\left(c(v) r_{x}+h(v)\right)-a^{\prime}(v) v_{x}\left(c(v) r_{x}\right)^{2} \\
& +\frac{1}{2} h^{\prime}(v)\left(1+(\gamma+1) v^{-1} r_{x}\right) v_{x} .
\end{aligned}
$$

By $(1.1)_{2},(2.12)$, and $(2.15)$, we are able to show that

$$
\begin{aligned}
& s_{x}(0, t)=r_{x}(0, t)-\frac{2}{\sqrt{\gamma}}(v(0, t))^{(\gamma+1) / 2}\left(u_{1}(t)+u_{1}^{\prime}(t)\right), \\
& r_{x}(1, t)=s_{x}(1, t)-\frac{2}{\sqrt{\gamma}}(v(1, t))^{(\gamma+1) / 2}\left(u_{2}(t)+u_{2}^{\prime}(t)\right) .
\end{aligned}
$$

In terms of $(2.12),(2.20),(2.42)$, and the assumptions of Theorem 2.1, we are able to show that

$$
\lim _{t \rightarrow 0}\left|\left(r_{x}, s_{x}\right)(0, t)\right| \leq O(1)\left(N_{1}+\mu_{0}+\delta_{0}\right)
$$


Differentiating $(1.1)_{2}$ with respect to $t$, we get, in terms of $(1.1)_{1},(2.12),(2.42)$, and (2.43), that

$$
\begin{aligned}
s_{x x}(0, t) & =-r_{x x}(0, t)-\frac{2}{\gamma}(v(0, t))^{(\gamma+1)}\left(u_{1}^{\prime \prime}(t)+u_{1}^{\prime}(t)\right)+2(\gamma+1) v^{-1} v_{x} u_{x}(0, t) \\
& =:-r_{x x}(0, t)+g_{1}(t), \\
r_{x x}(1, t) & =-s_{x x}(1, t)-\frac{2}{\gamma}(v(1, t))^{(\gamma+1)}\left(u_{2}^{\prime \prime}(t)+u_{2}^{\prime}(t)\right)+2(\gamma+1) v^{-1} v_{x} u_{x}(1, t) \\
& =:-s_{x x}(1, t)+g_{2}(t) .
\end{aligned}
$$

With the help of (2.42)-(2.43) and (2.45)-(2.46), we can obtain the boundary conditions for $Y_{2}$ and $Z_{2}$, respectively, as follows.

At $x=0$, it follows that

$$
\begin{aligned}
Y_{2}(0, t) & =c(v) s_{x x}(0, t)+c(v)_{x} s_{x}(0, t)+h(v)_{x}(0, t) \\
& =-c(v) r_{x x}(0, t)+c(v)(0, t) g_{1}(t)+c(v)_{x} s_{x}(0, t)+h(v)_{x}(0, t) \\
& =-Z_{2}(0, t)+2 c(v)_{x} u_{x}(0, t)+2 h(v)_{x}(0, t)+c(v)(0, t) g_{1}(t) \\
& =:-Z_{2}(0, t)+g_{3}(t),
\end{aligned}
$$

and at $x=1$, it follows that

$$
\begin{aligned}
Z_{2}(1, t) & =c(v) r_{x x}(1, t)+c(v)_{x} r_{x}(1, t)+h(v)_{x}(1, t) \\
& =-c(v) s_{x x}(1, t)+c(v)(1, t) g_{2}(t)+c(v)_{x} r_{x}(1, t)+h(v)_{x}(1, t) \\
& =-Y_{2}(1, t)+2 c(v)_{x} u_{x}(1, t)+2 h(v)_{x}(1, t)+c(v)(1, t) g_{2}(t) \\
& =:-Y_{2}(1, t)+g_{4}(t) .
\end{aligned}
$$

Lemma 2.7. Assume that the piecewise smooth solution of the IBVP (1.1), (1.3), (1.4), and (2.3) exists on $\Omega\left(T_{1}\right)$. Then, under the assumptions of Theorem 2.1, it follows that on $\Omega_{s}\left(T_{1}\right)$

$$
\left|s_{x x}\right|+\left|r_{x x}\right|+\left|s_{x x x}\right|+\left|r_{x x x}\right| \leq C_{0},
$$

with $C_{0}>0$ a constant, provided that (2.17) on $\Omega_{s}\left(T_{1}\right)$ and (2.19) hold.

Proof. We estimate the terms in the left-hand side of $(2.49)$ on $\Omega_{2}^{+}\left(0, T_{1}\right)$ and $\Omega_{2}^{-}\left(0, T_{1}\right)$, respectively. On $\Omega_{2}^{+}\left(0, T_{1}\right)$, the bounds of $\left|s_{x x}\right|,\left|r_{x x}\right|,\left|s_{x x x}\right|$, and $\left|r_{x x x}\right|$ can be obtained by solving the corresponding initial boundary value problems for them. In fact, on $\Omega_{2}^{+}\left(0, T_{1}\right)$ the systems $(2.36)$ and $(2.37)$ are linear for $\left(Z_{2}, Y_{2}\right)$. Integrating $(2.36)$ and (2.37) along 1 -characteristics and 2-characteristics, respectively on $[0, t]$, and noticing, due to (2.17), that

$$
b_{3}(x, t) \geq \frac{1}{4}, \quad b_{4}(x, t) \geq \frac{1}{4}, \quad(x, t) \in \Omega_{2}^{+}\left(0, T_{1}\right),
$$

and

$$
\left|f_{5}(x, t)\right|+\left|f_{6}(x, t)\right| \leq O(1)\left|v_{x}(x, t)\right|, \quad(x, t) \in \Omega_{2}^{+}\left(0, T_{1}\right),
$$

we can show, for any $(x, t) \in \Omega_{2}^{+}\left(0, T_{1}\right)$, that

$$
\left|Y_{2}(x, t)\right|+\left|Z_{2}(x, t)\right| \leq O(1)\left(N_{1}+\mu_{0}+\max _{(x, t) \in \Omega_{2}^{+}\left(0, T_{1}\right)}\left|v_{x}(x, t)\right|\right),
$$


where we have used (2.48). Then, by $(2.12)$, we gain, for any $(x, t) \in \Omega_{2}^{+}\left(0, T_{1}\right)$, that

$$
\left|s_{x x}\right|+\left|r_{x x}\right| \leq O(1)\left(N_{1}+\mu_{0}+\max _{(x, t) \in \Omega_{2}^{+}\left(0, T_{1}\right)}\left|v_{x}(x, t)\right|\right) .
$$

Similarly, we can yield the corresponding IBVP for $\left(Y_{2}\right)_{x}$ and $\left(Z_{2}\right)_{x}$ on $\Omega_{2}^{+}\left(0, T_{1}\right)$ and then obtain, for any $(x, t) \in \Omega_{2}^{+}\left(0, T_{1}\right)$, that

$$
\left|s_{x x x}\right|+\left|r_{x x x}\right| \leq O(1)\left(N_{2}+N_{1}+\mu_{0}+\max _{(x, t) \in \Omega_{2}^{+}\left(0, T_{1}\right)}\left|v_{x}(x, t)\right|\right) .
$$

Now, we estimate them on $\Omega_{2}^{-}\left(0, T_{1}\right)$. Differentiating $(2.20)$ with respect to $t$, we have, after a careful calculation, that along $x=x_{2}(t)$,

$$
\begin{aligned}
a_{r}^{-}(1 & \left.+J_{2}^{-}\right) \mu_{2}^{-}\left(r^{-}\right)_{x x}-a_{s}^{-}\left(1-J_{2}^{-}\right) \mu_{2}^{-}\left(s^{-}\right)_{x x} \\
& =a_{r}^{+}\left(1+J_{2}^{+}\right) \mu_{2}^{+}\left(r^{+}\right)_{x x}-a_{s}^{+}\left(1-J_{2}^{+}\right) \mu_{2}^{+}\left(s^{+}\right)_{x x}+h_{1}(t) \\
& =: h_{2}(t),
\end{aligned}
$$

where

$$
\begin{aligned}
h_{1}(t)= & -a_{r}^{-}\left\{\left(\mu_{2}^{-}\right)_{x}\left(r^{-}\right)_{x}-\frac{1}{2}\left(\left(r^{-}\right)_{x}+\left(s^{-}\right)_{x}\right)\right\}+a_{s}^{-}\left\{\left(\mu_{2}^{-}\right)_{x}\left(s^{-}\right)_{x}+\frac{1}{2}\left(\left(r^{-}\right)_{x}+\left(s^{-}\right)_{x}\right)\right\} \\
& +a_{r}^{+}\left\{\left(\mu_{2}^{+}\right)_{x}\left(r^{+}\right)_{x}-\frac{1}{2}\left(\left(r^{+}\right)_{x}+\left(s^{+}\right)_{x}\right)\right\}-a_{s}^{+}\left\{\left(\mu_{2}^{+}\right)_{x}\left(s^{+}\right)_{x}+\frac{1}{2}\left(\left(r^{+}\right)_{x}+\left(s^{+}\right)_{x}\right)\right\} \\
& -\left(r^{-}\right)_{x} D_{t}^{+} a_{r}^{-}-\left(s^{-}\right)_{x} D_{t}^{+} a_{s}^{-}+\left(r^{+}\right)_{x} D_{t}^{+} a_{r}^{+}+\left(s^{+}\right)_{x} D_{t}^{+} a_{s}^{+} \\
& +2 \sqrt{\gamma} D_{t}^{+}\left(J_{2}^{-}[v]_{2}\right) .
\end{aligned}
$$

It follows from (2.54) that

$$
\begin{aligned}
a_{r}^{-}(1+ & \left.J_{2}^{-}\right) \mu_{2}^{-} Z_{2}-a_{s}^{-}\left(1-J_{2}^{-}\right) \mu_{2}^{-} Y_{2} \\
= & a_{r}^{-}\left(1+J_{2}^{-}\right) \mu_{2}^{-}\left(c\left(v^{-}\right)_{x}\left(r^{-}\right)_{x}+h\left(v^{-}\right)_{x}\right) \\
& -a_{s}^{-}\left(1-J_{2}^{-}\right) \mu_{2}^{-}\left(c\left(v^{-}\right)_{x}\left(s^{-}\right)_{x}+h\left(v^{-}\right)_{x}\right)+c\left(v^{-}\right) h_{2}(t) \\
= & : h_{3}(t) .
\end{aligned}
$$

By (2.44) and the assumptions of Theorem 2.1, we find that

$$
\lim _{t \rightarrow 0}\left(\left|g_{3}(t)\right|+\left|h_{3}(t)\right|\right) \leq O(1)\left(N_{1}+\mu_{0}+\delta_{0}\right) .
$$

Thus, we have, in terms of (2.44), (2.47), (2.55), (2.56), and the assumptions of Theorem 2.1 , that

$$
\lim _{t \rightarrow 0}\left(\left|\left(Z_{2}, Y_{2}\right)(0, t)\right|\right) \leq O(1)\left(N_{1}+\mu_{0}+\delta_{0}\right)
$$

Noticing

$$
\left|h_{3}(t)\right| \leq O(1)\left(N_{1}+\left|[v]_{2}\right|+\max _{(x, t) \in \Omega_{s}\left(T_{1}\right)}\left|v_{x}(x, t)\right|\right), \quad 0 \leq t \leq T_{1},
$$

and using Lemma 2.6, (2.36), (2.37), (2.47), (2.55), and (2.57), we can estimate the bounds of $\left|Y_{2}\right|,\left|Z_{2}\right|$ on $\Omega_{2}^{-}\left(0, T_{1}\right)$ with $t>0$ and obtain, for any $(x, t) \in \Omega_{2}^{-}\left(0, T_{1}\right)$ with $t>0$, that

$$
\left|s_{x x}\right|+\left|r_{x x}\right| \leq O(1)\left(N_{1}+\mu_{0}+\delta_{0}+\max _{(x, t) \in \Omega_{s}\left(T_{1}\right)}\left|v_{x}(x, t)\right|\right),
$$


provided that (2.17) and (2.19) hold. Similarly, we can obtain, for $(x, t) \in \Omega_{2}^{-}\left(0, T_{1}\right)$ with $t>0$, that

$$
\left|s_{x x x}\right|+\left|r_{x x x}\right| \leq O(1)\left(N_{2}+N_{1}+\mu_{0}+\delta_{0}+\max _{(x, t) \in \Omega_{s}\left(T_{1}\right)}\left|v_{x}(x, t)\right|\right) .
$$

Thus, the proof is completed.

LEMmA 2.8. Under the assumption of Theorem 2.1, it follows that, along $x=x_{2}(t)$,

$$
\left|\left[s_{x}\right]_{2}\right|+\left|\left[r_{x}\right]_{2}\right|+\left|\left[s_{x x}\right]_{2}\right|+\left|\left[r_{x x}\right]_{2}\right| \leq O(1) \delta_{0} e^{-B_{3} t}, \quad 0 \leq t<T_{1},
$$

provided that (2.17) on $\Omega_{s}\left(T_{1}\right)$ and (2.19) hold.

Proof. We first estimate $\left[s_{x}\right]_{2}$. Due to Lemma 2.6 and

$$
\left|\left[s_{x}\right]_{2}\right| \leq O(1)\left(\left|\left[Y_{1}\right]_{2}\right|+\left|[v]_{2}\right|\right),
$$

we only need to estimate $\left[Y_{1}\right]_{2}$. By $(2.32)$ it follows, along $x=x_{2}(t)$, that

$$
\begin{aligned}
D_{t}^{+}\left[Y_{1}\right]_{2}= & \left(\dot{x}_{2}-\mu_{2}^{+}\right)\left(Y_{1}^{+}\right)_{x}-\left(\dot{x}_{2}-\mu_{2}^{-}\right)\left(Y_{1}^{-}\right)_{x} \\
& -[a]_{2}\left(Y_{1}^{+}\right)^{2}-a^{-}\left[Y_{1}^{-}\right]_{2}-\left[b_{1}\right]_{2} Y_{1}^{+}-b_{1}^{-}\left[Y_{1}\right]_{2}+\left[f_{3}\right]_{2} \\
= & :-b_{5}(t)\left[Y_{1}\right]_{2}+f_{7}(t),
\end{aligned}
$$

where

$$
\begin{aligned}
b_{5}(t)= & \frac{1}{2}+a^{-}[h]_{2}+a^{-} c^{-}\left(s^{-}\right)_{x}+a^{-} c^{+}\left(s^{+}\right)_{x} \\
f_{7}(t)= & \left(\dot{x}_{2}-\mu_{2}^{+}\right)\left(Y_{1}^{+}\right)_{x}-\left(\dot{x}_{2}-\mu_{2}^{-}\right)\left(Y_{1}^{-}\right)_{x} \\
& -[a]_{2}\left(Y_{1}^{+}\right)^{2}-\left[b_{1}\right]_{2} Y_{1}^{+}+\left[f_{3}\right]_{2} .
\end{aligned}
$$

By (2.27), (2.28), and Lemma 2.6, we have

$$
b_{5}(t) \geq \frac{1}{4}
$$

and

$$
\left|[h]_{2}\right|+\left|[a]_{2}\right|+\left|\left[b_{1}\right]_{2}\right|+\left|\left[f_{3}\right]_{2}\right|+\left|\dot{x}-\mu_{2}^{ \pm}\right| \leq O(1) \delta_{0} e^{-B_{1} t},
$$

provided that $(2.17)$ on $\Omega_{s}\left(T_{1}\right)$ and $(2.19)$ hold. Then, it follows from (2.65) and Lemma 2.7 that

$$
\left|f_{7}(t)\right| \leq O(1) \delta_{0} e^{-B_{1} t}
$$

Multiplying (2.60) by $e^{\int_{0}^{t} b_{5}(\tau) d \tau}$ and integrating it over $[0, t]$, we have, in terms of $(2.64)$ and (2.66), that

$$
\left|\left[Y_{1}\right]_{2}\right| \leq O(1) \delta_{0} e^{-B_{3} t}
$$

where

$$
B_{3}= \begin{cases}\frac{1}{4}, & \text { if } B_{1}>\frac{1}{4} \\ B_{1}, & \text { if } B_{1}<\frac{1}{4} \\ \frac{1}{5}, & \text { otherwise }\end{cases}
$$

Similarly, we can estimate the other terms in the left-hand side of (2.59). 
2.3. Energy estimates. In this subsection, we estimate the bounds of $v, v_{x}$, and $u_{x}$ on $\Omega_{s}\left(T_{1}\right)$. By substituting $(1.1)_{1}$ into $(1.1)_{2}$, the IBVP $(1.1),(1.3)$, and (1.4) can be reformulated into

$$
\begin{aligned}
& L(v) \equiv v_{t t}+p(v)_{x x}+v_{t}=0, \quad(x, t) \in \Omega_{s}\left(T_{1}\right), \\
& p(v(0, t))_{x}=-f_{1}, \quad p(v(1, t))_{x}=-f_{2}, \quad 0 \leq t \leq T_{1}, \\
& v(x, 0)=v_{0}, \quad v_{t}(x, 0)=u_{0 x}, \quad x \in(0,1) .
\end{aligned}
$$

For any $(x, t) \in \Omega_{s}\left(T_{1}\right)$, consider the following equality:

$$
\left(v-\bar{v}_{1}+2 v_{t}\right) L(v)=0 .
$$

Integrating (2.69) over $\Omega_{s}(t)$, we get, after a computation, that

$$
\frac{1}{2} E_{1}(t) d x+\int_{0}^{t} E_{2}(\tau) d \tau=\frac{1}{2} E_{0}+G_{2}(t)+\int_{0}^{t} G_{1}(\tau) d \tau
$$

where

$$
\begin{aligned}
E_{1}(t) \equiv & \int_{D}\left\{\left(v-\bar{v}_{1}\right)^{2}+2\left(v-\bar{v}_{1}\right) v_{t}+2 v_{t}^{2}+2 \gamma v^{-(\gamma+1)} v_{x}^{2}\right\}(x, t) d x \\
E_{2}(t) \equiv & \int_{D}\left\{v_{t}^{2}+\gamma v^{-(\gamma+1)}\left(1+(\gamma+1) v^{-1} v_{t}\right) v_{x}^{2}\right\}(x, t) d x \\
E_{0}= & \int_{0}^{1}\left\{\left(v_{0}-\bar{v}_{1}\right)^{2}+2\left(v_{0}-\bar{v}_{1}\right) u_{0 x}^{2}+2 \gamma v_{0}^{-(\gamma+1)} v_{0 x}^{2}\right\} d x \\
G_{2}(t) \equiv & \int_{0}^{t}\left\{f_{2}(\tau)\left(2 v_{t}+\left(v-\bar{v}_{1}\right)\right)(1, \tau)-f_{1}(\tau)\left(2 v_{t}+\left(v-\bar{v}_{1}\right)\right)(0, \tau)\right\} d \tau \\
G_{1}(t) \equiv & -\frac{1}{2} \dot{x}_{2}(t)\left[\left(v-\bar{v}_{1}\right)^{2}+2\left(v-\bar{v}_{1}\right) v_{t}+2 v_{t}^{2}+2 \gamma v^{-(\gamma+1)} v_{x}^{2}\right]_{2} \\
& +\left[\left(v-\bar{v}_{1}+2 v_{t}\right) p(v)_{x}\right]_{2},
\end{aligned}
$$

with

$$
\int_{D} f(x) d x=\left\{\int_{0}^{x_{2}(t)-}+\int_{x_{2}(t)+}^{1}\right\} f(x) d x .
$$

By (1.1) and (2.12), we can verify that

$$
\left|G_{1}(t)\right| \leq C\left(\left|[v]_{2}\right|+\left|\left[r_{x}\right]_{2}\right|+\left|\left[s_{x}\right]_{2}\right|\right),
$$

provided that $(2.17)$ holds.

By (1.8) and (1.13), we get, for $(x, t) \in \Omega_{s}(t)$, that

$$
\left|v-\bar{v}_{1}\right|^{2} \leq C\left(|g(t)|^{2}+2\left\|v_{x}\right\|^{2}+\left|[v]_{2}\right|^{2}\right) .
$$

Noticing, for $(x, t) \in \Omega_{s}(t)$, that

$$
\left|v_{t}\right|^{2} \leq C\left(\left\|\left(v_{t}, v_{x t}\right)\right\|^{2}+\left|\left[u_{x}\right]_{2}\right|^{2}\right),
$$

we obtain, with the help of (2.69)-(2.74), the Cauchy inequality, Lemma 2.6, and Lemma 2.8 , the following lemma. 
LEMmA 2.9. Under the assumptions of Theorem 2.1, it follows that

$$
\begin{aligned}
\|(v- & \left.\bar{v}_{1}, u_{x}, \sqrt{\gamma} v^{-(\gamma+1) / 2} v_{x}\right)(\cdot, t)\left\|^{2}+\int_{0}^{t}\right\|\left(u_{x}, \sqrt{\gamma} v^{-(\gamma+1) / 2} v_{x}\right)(\cdot, \tau) \|^{2} d \tau \\
& \leq C\left(N_{1}+\mu_{0}+\delta_{0}\right)+\frac{1}{16} \int_{0}^{t}\left\|\sqrt{\gamma} v^{-(\gamma+1) / 2} v_{x t}(\cdot, \tau)\right\|^{2} d \tau, \quad t \in\left[0, T_{1}\right],
\end{aligned}
$$

provided that $(2.17)$ on $\Omega_{s}\left(T_{1}\right)$ and $(2.19)$ hold, where and from now on $C$ is a generic positive constant.

Similarly, for any $(x, t) \in \Omega_{s}(t)$, consider the following equality:

$$
\left(v_{t}+2 v_{t t}\right) \partial_{t} L(v)=0
$$

Integrating $(2.75)$ over $\Omega_{s}(t)$, we obtain

$$
\frac{1}{2} E_{3}(t)+\int_{0}^{t}\left(E_{4}+E_{5}\right)(\tau) d \tau=\frac{1}{2} E_{6}+G_{4}(t)+\int_{0}^{t} G_{3}(\tau) d \tau
$$

where

$$
\begin{aligned}
E_{3}(t) \equiv & \int_{D}\left\{v_{t}^{2}+2 v_{t} v_{t t}+2 v_{t t}^{2}+2 \gamma v^{-(\gamma+1)} v_{x t}^{2}\right. \\
& \left.-4 \gamma(\gamma+1) v^{-(\gamma+2)} v_{x} v_{t} v_{x t}\right\}(x, t) d x \\
E_{4}(t) \equiv & \int_{D}\left\{v_{t t}^{2}+\gamma v^{-(\gamma+1)}\left(1+3(\gamma+1) v^{-1} v_{t}\right) v_{x t}^{2}\right\}(x, t) d x \\
E_{5}(t) \equiv & \int_{D}\left\{2 \gamma(\gamma+1) v^{-(\gamma+2)} v_{x} v_{t t}-2 \gamma(\gamma+1)(\gamma+2) v^{-(\gamma+3)} v_{x} v_{t}^{2}\right. \\
E_{6} \equiv & \int_{0}^{1}\left\{u_{0 x}^{2}-2 u_{0 x}\left(u_{0 x}+p\left(v_{0}\right)_{x x}\right)+2\left(u_{0 x}+p\left(v_{0}\right) x\right)^{2}+2 \gamma v_{0}^{-(\gamma+1)} u_{0 x x}^{2}\right. \\
& -\int_{0}^{t}\left\{2 f_{2 t t}(\tau) u_{t}(1, \tau)-2 f_{1 t t}(\tau) v_{t}(0, \tau)\right\} d \tau \\
& +2 f_{2 t}(\tau) v_{t}(1, \tau)-2 f_{1 t}(0) v_{t}(1,0)-2 f_{2 t}(\tau) v_{t}(0, \tau)+2 f_{1 t}(0) v_{t}(0,0) \\
G_{4}(t)= & \int_{0}^{t}\left\{f_{2 t}(\tau) v_{t}(1, \tau)-f_{1 t}(\tau) v_{t}(0, \tau)\right\} d \tau \\
& +\frac{1}{2} \dot{x}(t)\left[\left(v_{t}^{2}+2 v_{t} v_{t t}+2 v_{t t}^{2}+2 \gamma v^{-(\gamma+1)} v_{x t}^{2}-4 \gamma(\gamma+1) v^{-(\gamma+2)} v_{x} v_{t} v_{x t}\right)\right]_{2} \\
& +\left[\left(v_{t}+2 v_{t t}\right) p_{x t}\right]_{2} .
\end{aligned}
$$

By $(2.44),(2.74)$, and the Cauchy inequality, we can estimate $G_{4}(t)$ as follows:

$$
\begin{aligned}
\left|G_{4}(t)\right| \leq & C\left(\mu_{0}+N_{1}\right)+\frac{1}{4}\left\|\sqrt{\gamma} v^{-(\gamma+1) / 2} v_{x}\right\|^{2} \\
& +\frac{1}{16} \int_{0}^{t}\left\|\left(u_{x}, \sqrt{\gamma} v^{-(\gamma+1) / 2} v_{x t}\right)\right\|^{2} d \tau .
\end{aligned}
$$


The $G_{3}(t)$ can be estimated, in terms of Lemma $2.7,(1.1)$, and $(2.12)$, as

$$
\left|G_{3}(t)\right| \leq C\left(\left|[v]_{2}\right|+\left|\left[v_{x}\right]_{2}\right|+\left|\left[u_{x}\right]_{2}\right|+\left|\left[v_{x x}\right]_{2}\right|+\left|\left[u_{x x}\right]_{2}\right|\right),
$$

provided that (2.17) holds.

It is easy to verify that

$$
\begin{aligned}
& \left|\int_{0}^{t} E_{5}(\tau) d \tau\right| \\
& \leq \int_{0}^{t} \int_{D} \gamma v^{-(\gamma+1)}\left(\frac{5}{24}+2 \gamma(\gamma+1)^{2} v^{-(\gamma+2)} v_{x}^{2}\right) v_{x t}^{2} d x d \tau \\
& +\int_{0}^{t} \int_{D}\left\{\frac{1}{2} v_{t t}^{2}+\gamma v^{-(\gamma+1)} v_{x}^{2}\left(\frac{1}{24}+\frac{(\gamma+2)^{2}}{54(\gamma+1)^{2}}\right)\right\} d x d \tau \\
& \leq \frac{1}{2} \int_{0}^{t}\left\|\left(v_{t t}, \sqrt{\frac{1}{2}} \gamma v^{-(\gamma+1) / 2} v_{x t}\right)\right\|^{2} d \tau+\frac{1}{12} \int_{0}^{t}\left\|\sqrt{\gamma} v^{-(\gamma+1) / 2} v_{x}\right\|^{2} d \tau,
\end{aligned}
$$

provided that (2.17) holds.

Then, we have, with the help of (2.78)-(2.80), Lemma 2.6, Lemma 2.8, and $(2.68)_{1}$, the following lemma.

LEMmA 2.10. Under the assumptions of Theorem 2.1, it follows that

$$
\begin{gathered}
\left\|\left(v_{t}, u_{x t}, \sqrt{\gamma} v^{-(\gamma+1) / 2} v_{x t}\right)(\cdot, t)\right\|^{2}+\int_{0}^{t}\left\|\left(u_{x t}, \sqrt{\gamma} v^{-(\gamma+1) / 2} v_{x t}, v_{x x}\right)(\cdot, \tau)\right\|^{2} d \tau \\
\leq C\left(N_{1}+\mu_{0}+\delta_{0}\right), \quad t \in\left[0, T_{1}\right]
\end{gathered}
$$

provided that (2.17) on $\Omega_{s}\left(T_{1}\right)$ and (2.19) hold.

Noticing that, for $(x, t) \in \Omega_{s}\left(T_{1}\right)$, it follows that

$$
|u(x, t)| \leq O(1)\left(\left|u_{1}(t)\right|+\left|u_{2}(t)\right|+\left\|u_{x}(\cdot, t)\right\|+\left|[v]_{2}\right|\right),
$$

we have, by $(2.68)_{1},(2.81)$, and Lemmas $2.9-2.10$, the following lemma.

LEmma 2.11. Under the assumptions of Theorem 2.1, it follows, for $t \in\left[0, T_{1}\right]$, that

$$
\left\|\left(v-\bar{v}_{1}\right)(\cdot, t)\right\|_{2}^{2}+\|u(\cdot, t)\|_{2}^{2}+\int_{0}^{t}\left\|\left(u_{x}, u_{x t}, v_{x}, v_{x t}, v_{x x}\right)(\cdot, \tau)\right\|^{2} d \tau \leq C\left(N_{1}+\mu_{0}+\delta_{0}\right),
$$

provided that $(2.17)$ on $\Omega_{s}\left(T_{1}\right)$ and $(2.19)$ hold.

Thus, with the help of Lemma 2.6 and Lemma 2.11, we can prove that (2.17) on $\Omega_{s}\left(T_{1}\right)$ and (2.19) really hold if we choose the initial-boundary data and the initial jump small enough such that $C\left(N_{1}+\mu_{0}+\delta_{0}\right)<\frac{1}{16} \eta$. Therefore, by the standard continuity argument, we can prove the existence of piecewise smooth solutions for the IBVP (1.1), (1.3), (1.4), and (2.3) on $\Omega\left(T_{1}\right)$, such that $v$ is uniformly bounded, $\left|v_{x}\right|+\left|u_{x}\right| \ll 1$ for $(x, t) \in \Omega_{s}\left(T_{1}\right)$ with $x_{2}\left(T_{1}\right)<1$, and $\left|[v]_{2}\right| \ll 1$, under the assumptions of Theorem 2.1. 
2.4. Global existence of the weak entropy solution. Now, we turn to prove the global existence of a piecewise smooth solution for $\operatorname{IBVP}(1.1)$ on $\Omega(T)$ for any $T \geq T_{1}$. Due to the above argument, we may assume $x_{2}\left(T_{1}\right)=1$. Then, it follows from $(2.2)$, the continuity of $u_{2}(t)$, and Lemma 2.6 that $u\left(x_{2}\left(T_{1}\right)-0, T_{1}\right)>u_{2}\left(T_{1}\right)$. Thus, by a procedure similar to that used in the above, we are able to show that there is a $\widehat{T}_{2}>T_{1}$, such that the IBVP (1.1), (1.3), and (2.82),

$$
\left.(v, u)(x, t)\right|_{t=T_{1}}=(v, u)\left(x, T_{1}\right), \quad u\left(1-0, T_{1}\right)>u_{2}\left(T_{1}\right)
$$

has a unique weak entropy solution $(r, s)$ on the domain

$$
\Omega\left(T_{1}, \widehat{T}_{2}\right)=:\left\{(x, t) \mid T_{1} \leq t \leq \widehat{T}_{2}, 0 \leq x \leq 1\right\}
$$

in the class of piecewise smooth functions, which contains only a 1-shock $x=x_{1}(t)$ with $x_{1}\left(T_{1}\right)=1$. Moreover, the specific volume is bounded, $\left|\left(r_{x}, s_{x}\right)\right| \ll 1$ for $x \neq x_{1}(t)$, and $\left|[v]_{1}\right| \ll 1$ under the assumptions of Theorem 2.1. Thus, we can show that the piecewise smooth solution to the IBVP (1.1), (1.3), (1.4), and (2.3) exists on $\Omega\left(T_{2}\right)$ with $T_{2} \geq \widehat{T}_{2}$ and $x_{2}\left(T_{2}\right)>0$, and is smooth on the following domain:

$$
\Omega_{s}\left(T_{2}\right)=\Omega_{s}\left(T_{1}\right) \cup \Omega_{2}^{+}\left(T_{1}, T_{2}\right) \cup \Omega_{2}^{-}\left(T_{1}, T_{2}\right)
$$

Define

$$
\begin{aligned}
& K_{i}=\left(v_{1}^{-}\right)^{(\gamma+1) / 2}+(-1)^{i}\left(v_{1}^{+}\right)^{(\gamma+1) / 2}+(-1)^{i+1} \frac{1}{2} \sqrt{\gamma} \dot{x}_{1}[v]_{1}, \quad i=1,2, \\
& A_{1}=\sqrt{\gamma}-\frac{1}{2 \sqrt{\gamma}}\left(\left(\mu_{1}^{+}\right)^{2}+\left(\dot{x}_{1}\right)^{2}\right) \frac{\mu_{1}^{+}}{\dot{x}_{1}}, \quad J_{1}^{ \pm}=\frac{\dot{x}_{1}}{\mu_{1}^{ \pm}}, \\
& b_{s}^{-}=\left(1-J_{1}^{-}\right)\left(A_{1}-\mu_{1}^{-} K_{1}\right), \quad b_{s}^{+}=A_{1}\left(1-J_{1}^{+}\right), \\
& b_{r}^{-}=\left(1+J_{1}^{-}\right)\left(A_{1}-\mu_{1}^{-} K_{1}\right), \quad b_{r}^{+}=\left(1+J_{1}^{+}\right)\left(A_{1}-2 \mu_{1}^{+}\right),
\end{aligned}
$$

where $\mu_{1}^{ \pm}=\mu\left(v\left(x_{1}(t) \pm 0, t\right)\right)$.

Differentiating $(2.1)_{2}$ with respect to $t$, and using $(2.12)$, we have, after a careful analysis, the following lemma.

LEMma 2.12. Assume that the piecewise smooth solution of the IBVP (1.1), (1.3), (1.4), and (2.3) exists on $\Omega\left(T_{2}\right)$. Then, along $x=x_{1}(t)$, it follows that

$$
b_{s}^{+}\left(s_{1}^{+}\right)_{x}+b_{r}^{+}\left(r_{1}^{+}\right)_{x}=b_{s}^{-}\left(s_{1}^{-}\right)_{x}+b_{r}^{-}\left(r_{1}^{-}\right)_{x}+2 \sqrt{\gamma} J_{1}^{+}[v]_{1}
$$


and

$$
\begin{aligned}
& -\frac{\left\{2 \sqrt{-\gamma[p(v)]_{1}[v]_{1}}+\left([p(v)]_{1}-\gamma\left(v_{1}^{+}\right)^{-(\gamma+1)}[v]_{1}\right)\left(v_{1}^{+}\right)^{-(\gamma+1) / 2}\right\}}{[v]_{1} \sqrt{-[p(v)]_{1}[v]_{1}}} D_{t}^{-}[v]_{1} \\
& =2\left(v_{1}^{+}\right)^{(\gamma+1) / 2} \sqrt{-[p(v)]_{1} /[v]_{1}} \\
& -\frac{2\left(\sqrt{\gamma}\left(v_{1}^{+}\right)^{-(\gamma+1) / 2}-\sqrt{-[p(v)]_{1} /[v]_{1}}\right)}{[v]_{1}}\left(r_{1}^{+}\right)_{x} \\
& +\frac{\left(\sqrt{\gamma}\left(v_{1}^{-}\right)^{-(\gamma+1) / 2}+\sqrt{-[p(v)]_{1} /[v]_{1}}\right) K_{1}}{[v]_{1}}\left(s_{1}^{-}\right)_{x} \\
& -\frac{\left(-\sqrt{\gamma}\left(v_{1}^{-}\right)^{-(\gamma+1) / 2}+\sqrt{-[p(v)]_{1} /[v]_{1}}\right) K_{2}}{[v]_{1}}\left(r_{1}^{-}\right)_{x}
\end{aligned}
$$

where $D_{t}^{-}=: \partial_{t}+\dot{x}_{1}(t) \partial_{x}$.

Define

$$
\delta_{1}=v\left(x_{1}\left(T_{1}\right)-0, T_{1}\right)-v\left(x_{1}\left(T_{1}\right)+0, T_{1}\right) .
$$

Noticing the facts that, at the point $(x, t)=\left(1, T_{1}\right)$,

$$
\dot{x}_{1}\left(T_{1}\right)[u]_{1}=[v]_{1}, \quad \dot{x}_{1}\left(T_{1}\right)[u]_{2}=[v]_{2}, \quad v_{2}^{-}=v_{1}^{-}, \quad[u]_{1}=[u]_{2}
$$

hold, we can verify that, at the point $(x, t)=\left(1, T_{1}\right)$,

$$
\frac{\mu_{m}}{\mu_{M}} \delta_{0} e^{-B_{2} T_{1}} \leq \delta_{1} \leq \delta_{0} \frac{\mu_{M}}{\mu_{m}} e^{-B_{1} T_{1}} .
$$

Therefore, we obtain the following exponential decay for the shock strength along $x=$ $x_{1}(t)$, i.e., (2.88) and (2.89).

LEMmA 2.13. Assume that the piecewise smooth solution of the IBVP (1.1), (1.3), (1.4), and (2.3) exists on $\Omega\left(T_{2}\right)$. Then, along $x=x_{1}(t)$, it follows for $t \in\left[T_{1}, T_{2}\right]$ that

$$
\begin{gathered}
-\frac{1}{[v]_{1}} D_{t}^{-}\left([v]_{1}\right) \in\left[B_{1}, B_{2}\right], \\
\delta_{0} \frac{\mu_{m}}{\mu_{M}} e^{-B_{2} t} \leq \delta_{1} e^{-B_{2}\left(t-T_{1}\right)} \leq\left|[v]_{1}\right| \leq \delta_{1} e^{-B_{1}\left(t-T_{1}\right)} \leq \delta_{0} \frac{\mu_{M}}{\mu_{m}} e^{-B_{1} t},
\end{gathered}
$$

provided that $(2.17)$ holds on $\Omega_{s}\left(T_{2}\right)$ and the shock strength is small enough.

By a procedure similar to that used in proving Lemma 2.7 and Lemma 2.8 , the estimate (2.49) can also be proved to be true on $\Omega_{1}^{ \pm}\left(T_{1}, T_{2}\right)$ and the exponential decay of $\left|\left[s_{x}\right]_{1}\right|,\left|\left[r_{x}\right]_{1}\right|,\left|\left[s_{x x}\right]_{1}\right|$, and $\left|\left[r_{x x}\right]_{1}\right|$ can be obtained. Thus, we have, in terms of Lemma 2.7 and Lemma 2.13, the following lemma.

LEMmA 2.14. Assume that the piecewise smooth solution of the IBVP (1.1), (1.3), (1.4), and (2.3) exists on $\Omega\left(T_{2}\right)$. Then, under the assumptions of Theorem 2.1 , it follows that on $\Omega_{s}\left(T_{2}\right)$

$$
\left|s_{x x}\right|+\left|r_{x x}\right|+\left|s_{x x x}\right|+\left|r_{x x x}\right| \leq C_{0}
$$

and that, along $x=x_{1}(t)$

$$
\left|\left[s_{x}\right]_{1}\right|+\left|\left[r_{x}\right]_{1}\right|+\left|\left[s_{x x}\right]_{1}\right|+\left|\left[r_{x x}\right]_{1}\right| \leq O(1) \delta_{0} e^{-B_{3} t}, \quad T_{1} \leq t<T_{2},
$$


provided that (2.17) holds on $\Omega_{s}\left(T_{2}\right)$ and the shock strength is small enough.

In terms of Lemmas $2.13-2.14$ and by a procedure similar to that used in proving Lemmas 2.9-2.11, we can prove that the piecewise smooth solution of the IBVP (1.1), (1.3), (1.4), and (2.3) exists on $\Omega\left(T_{2}\right)$ such that $v$ is uniformly bounded, $\left|v_{x}\right|+\left|u_{x}\right| \ll 1$ for $(x, t) \in \Omega_{s}\left(T_{2}\right)$, and the shock strength decays exponentially. Therefore, we have proved the existence of the piecewise smooth solution to the IBVP (1.1), (1.3), (1.4), and (2.3) on $\Omega\left(T_{2}\right)$ with $x_{1}\left(T_{2}\right) \geq 0$. Repeating the above procedures, we can prove the existence of a piecewise smooth solution to the IBVP (1.1), (1.3), (1.4), and (2.3) on $\Omega(T)$ for any $T>0$. In fact, without loss of generality, we assume that there exist $T_{i} \geq 0$ $(i=0,1,2, \ldots, n)$ with $T_{i+1}>T_{i}, T_{2 n}=T$, and $T_{0}=0$, such that

$$
\Omega_{s}\left(T_{2 n}\right)=\bigcup_{i=0}^{n-1}\left(\Omega_{2}^{ \pm}\left(T_{2 i}, T_{2 i+1}\right) \cup \Omega_{1}^{ \pm}\left(T_{2 i+1}, T_{2 i+2}\right)\right) .
$$

Define, for $k=1,2, \ldots, n-1$,

$$
\delta_{i}= \begin{cases}\left|v\left(x_{2}\left(T_{i}+0\right)+0, T_{i}+0\right)-v\left(x_{2}\left(T_{i}+0\right)-0, T_{i}+0\right)\right|, & i=2 k, \\ \left|v\left(x_{1}\left(T_{i}+0\right)+0, T_{i}+0\right)-v\left(x_{1}\left(T_{i}+0\right)-0, T_{i}+0\right)\right|, & i=2 k+1 .\end{cases}
$$

Since at the reflection points $(x, t)=\left(0, T_{2 k}\right)$ and $(x, t)=\left(1, T_{2 k+1}\right), k=1,2, \ldots, n-1$, it holds that

$$
\begin{aligned}
\delta_{2 k+1} & =\left|\frac{\dot{x}_{2}\left(T_{2 k+1}\right)}{\dot{x}_{1}\left(T_{2 k+1}\right)}\right|\left|[v]_{2}\left(T_{2 k+1}\right)\right|, \\
\delta_{2 k} & =\left|\frac{\dot{x}_{1}\left(T_{2 k}\right)}{\dot{x}_{2}\left(T_{2 k}\right)}\right|\left|[v]_{1}\left(T_{2 k}\right)\right|,
\end{aligned}
$$

similarly to (2.87), we have, by Lemma 2.6 , Lemma $2.13,(2.1)$ and $(2.2)$, that

$$
\begin{aligned}
\frac{\mu_{m}}{\mu_{M}} \delta_{2 k} e^{-B_{2}\left(T_{2 k+1}-T_{2 k}\right)} & \leq \delta_{2 k+1} \leq \frac{\mu_{M}}{\mu_{m}} \delta_{2 k} e^{-B_{1}\left(T_{2 k+1}-T_{2 k}\right)}, \\
\frac{\mu_{m}}{\mu_{M}} \delta_{2 k-1} e^{-B_{2}\left(T_{2 k}-T_{2 k-1}\right)} & \leq \delta_{2 k} \leq \frac{\mu_{M}}{\mu_{m}} \delta_{2 k-1} e^{-B_{1}\left(T_{2 k}-T_{2 k-1}\right)} .
\end{aligned}
$$

Thus, it follows from (2.87), (2.95), (2.96), Lemma 2.6, and Lemma 2.13 that Lemma 2.15 holds.

LEmma 2.15. Assume that the piecewise smooth solution of the IBVP (1.1), (1.3), (1.4), and (2.3) exists on $\Omega(T)$. Then, under the assumptions of Theorem 2.1, along $x=x_{1}(t)$, it follows that for $k=0,1,2, \ldots, n-1$,

$$
\begin{gathered}
-\frac{1}{[v]_{2}} D_{t}^{-}\left([v]_{1}\right) \in\left[B_{1}, B_{2}\right], \quad t \in\left[T_{2 k+1}, T_{2 k+2}\right], \\
\delta_{0} e^{-B_{4} t} \leq\left|[v]_{1}\right| \leq \delta_{0} e^{-B_{5} t}, \quad t \in\left[T_{2 k+1}, T_{2 k+2}\right],
\end{gathered}
$$

and, along $x=x_{2}(t)$, it follows that for $k=0,1,2, \ldots, n-1$,

$$
\begin{array}{ll}
-\frac{1}{[v]_{2}} D_{t}^{+}\left([v]_{2}\right) \in\left[B_{1}, B_{2}\right], & t \in\left[T_{2 k}, T_{2 k+1}\right], \\
\delta_{0} e^{-B_{4} t} \leq[v]_{2} \leq \delta_{0} e^{-B_{5} t}, & t \in\left[T_{2 k}, T_{2 k+1}\right],
\end{array}
$$


for two positive constants $B_{4}$ and $B_{5}$, provided that $(2.17)$ holds on $\Omega_{s}(T)$ and the shock strength is small enough.

Denote the above continuous and piecewise smooth function by $x=x(t)$, namely, $x=x(t):[0, T] \rightarrow[0,1]$ such that $\dot{x}(t) \neq 0$ and

$$
\dot{x}(t)= \begin{cases}\dot{x}_{2}(t), & \text { if } \dot{x}(t)>0, \\ \dot{x}_{1}(t), & \text { if } \dot{x}(t)<0 .\end{cases}
$$

Let $[F](t)=F(x(t)+0, t)-F(x(t)-0, t)$. Then, repeating the same procedure, we have, in terms of Lemma 2.15, (2.95), (2.96) and (2.12), the following.

Lemma 2.16. Under the assumptions of Theorem 2.1, it follows, along $x=x(t)$, that

$$
\begin{aligned}
& \left|\left[v_{x}\right]\right|+\left|\left[v_{x x}\right]\right|+|[u]|+\left|\left[u_{x}\right]\right|+\left|\left[u_{x x}\right]\right| \leq O(1) \delta_{0} e^{-B_{6} t}, \quad t>0, \\
& \int_{0}^{T}\left(|[v]|+\left|\left[v_{x}\right]\right|+\left|\left[v_{x x}\right]\right|+|[u]|+\left|\left[u_{x}\right]\right|+\left|\left[u_{x x}\right]\right|\right) d t \leq O(1) \delta_{0},
\end{aligned}
$$

for some positive constant $B_{6}$, provided that (2.17) holds on $\Omega_{s}(T)$ and the shock strength is small enough.

With the help of Lemma 2.16, we can easily verify that Lemmas 2.9-2.11 hold uniformly for $0 \leq t \leq T$. Repeating the same procedure in proving the existence of a piecewise smooth solution for $0 \leq t \leq T_{1}$, we have the following global existence result.

TheOREM 2.17. Let $T>0$. Under the assumptions of Theorem 2.1, it follows that for $0<t<T$,

$$
\begin{array}{r}
\left\|v-\bar{v}_{1}\right\|_{2}^{2}+\|u\|_{2}^{2}+\int_{0}^{t}\left\|\left(u_{x}, u_{x t}, v_{x}, v_{x t}, v_{x x}\right)\right\|^{2} d \tau \leq C\left(N_{1}+\mu_{0}+\delta_{0}\right), \\
|[v]|+\left|\left[v_{x}\right]\right|+\left|\left[v_{x x}\right]\right|+|[u]|+\left|\left[u_{x}\right]\right|+\left|\left[u_{x x}\right]\right| \leq O(1) \delta_{0} e^{-B_{6} t}
\end{array}
$$

and for $x \neq x(t)$,

$$
\left|v_{x x}\right|+\left|u_{x x}\right| \leq C\left(N_{1}+\mu_{0}+\delta_{0}\right), \quad\left|v_{x x x}\right|+\left|u_{x x x}\right|<C .
$$

2.5. Asymptotic behavior of the weak entropy solution. To finish the proof of Theorem 2.1 , we only need to prove $(2.11)$, due to Theorem 2.17 .

Differentiating $[(2.69)+(2.75)]$ over $[0,1]$, we obtain

$$
\frac{1}{2} \frac{d}{d t}\left(E_{1}+E_{3}\right)+E_{2}+E_{4}+E_{5}=\frac{d}{d t}\left(G_{2}+G_{4}\right)+G_{5}(t)+G_{6}(t),
$$

where $E_{i}(i=1,2, \ldots, 5)$ are those in $(2.71)$ and $(2.77)$ with $x_{2}(t)$ replaced by $x(t)$, and

$$
\begin{aligned}
G_{5}(t) \equiv & -\frac{1}{2} \dot{x}(t)\left[\left(\left(v-\bar{v}_{1}\right)^{2}+2\left(v-\bar{v}_{1}\right) v_{t}+2 v_{t}^{2}+2 \gamma v^{-(\gamma+1)} v_{x}^{2}\right)\right] \\
& +\left[\left(v-\bar{v}_{1}+2 v_{t}\right) p(v)_{x}\right], \\
G_{6}(t) \equiv & -\frac{1}{2} \dot{x}(t)\left[\left(v_{t}^{2}+2 v_{t} v_{t t}+2 v_{t t}^{2}+2 \gamma v^{-(\gamma+1)} v_{x t}^{2}-4 \gamma(\gamma+1) v^{-(\gamma+2)} v_{x} v_{t} v_{x t}\right)\right] \\
& +\left[\left(v_{t}+2 v_{t t}\right) p_{x t}\right] .
\end{aligned}
$$


By Lemma 2.11, we find that

$$
\begin{aligned}
C^{-1} \| & \left(v-\bar{v}_{1}, u_{x}, u_{x t}, \sqrt{\gamma} v^{-(\gamma+1) / 2} v_{x}, \sqrt{\gamma} v^{-(\gamma+1) / 2} v_{x t}\right) \|^{2} \\
& \leq\left(E_{1}+E_{3}\right)(t) \\
& \leq C\left(E_{2}+E_{4}+E_{5}\right)(t)+C\left(|g(t)|^{2}+f_{9}(t)\right),
\end{aligned}
$$

where $f_{9}(t)=|[v]|^{2}+\left|\left[v_{x}\right]\right|^{2}+\left|\left[v_{x x}\right]\right|^{2}+\left|[u]_{x}\right|^{2}+\left|\left[u_{x x}\right]\right|^{2}$, and

$$
\begin{aligned}
\frac{d}{d t}\left(G_{2}+G_{4}\right)(t) \leq & \frac{1}{4}\left(E_{2}+E_{4}\right)+f_{2}(t) v_{t t}(1, t)-f_{1} v_{t t}(0, t) \\
& +C\left(f_{1}^{2}+f_{2}^{2}+f_{1 t}^{2}+f_{2 t}^{2}+g^{2}+f_{9}(t)\right)(t) .
\end{aligned}
$$

Thus, by (2.106) we get, with the help of (2.107), (2.108), and Theorem 2.17, that

$$
\begin{aligned}
\|(v- & \left.\bar{v}_{1}, u_{x}, u_{x t}, \sqrt{\gamma} v^{-(\gamma+1) / 2} v_{x}, \sqrt{\gamma} v^{-(\gamma+1) / 2} v_{x t}\right) \|^{2} \\
\leq & C e^{-\beta_{5} t}+C \int_{0}^{t}\left(f_{1}^{2}+f_{2}^{2}+f_{1 t}^{2}+f_{2 t}^{2}+g^{2}\right)(\tau) e^{-\beta_{5}(t-\tau)} d \tau \\
& +C \int_{0}^{t}\left(\left|f_{1}\right|+\left|f_{2}\right|+\left|f_{1 t}\right|+\left|f_{2 t}\right|\right)(\tau) e^{-\beta_{5}(t-\tau)} d \tau \\
& +C \int_{0}^{t}\left(\left|G_{5}(\tau)\right|+\left|G_{6}(\tau)\right|+f_{9}(t)\right) e^{-\beta_{5}(t-\tau)} d \tau
\end{aligned}
$$

for some positive constant $\beta_{5}$.

By (2.81), (2.109), Theorem 2.17, and the following Lemma 2.18, we can obtain (2.11). Thus, the proof of Theorem 2.1 is completed.

LEMma $2.18([22])$. Let $w(t)(t \geq 0)$ and $z(t)$ be continuous functions satisfying that there exist positive constants $c_{i}(i=1,2,3,4)$ such that for $0 \leq \tau \leq t$,

$$
c_{1} e^{c_{2}(t-\tau)} \leq \exp \left\{\int_{\tau}^{t} w(s) d s\right\} \leq c_{3} e^{c_{4}(t-\tau)} .
$$

Denote $F(t)$ by

$$
F(t)=\int_{t}^{t+1} z(\tau) d \tau
$$

Then

$$
\begin{aligned}
C^{-1} \underline{\lim }_{t \rightarrow+\infty} F(t) & \leq \underline{\lim }_{t \rightarrow+\infty} \int_{0}^{t} \exp \left\{-\int_{\tau}^{t} w(s) d s\right\} z(\tau) d \tau \\
& \leq \varlimsup_{t \rightarrow+\infty} \int_{0}^{t} \exp \left\{-\int_{\tau}^{t} w(s) d s\right\} z(\tau) d \tau \\
& \leq C \varlimsup_{t \rightarrow+\infty} F(t) .
\end{aligned}
$$

3. Two-shocks reflection. Let $u_{-}^{1}=u_{2}(0)$ and $u_{+}^{1}=\lim _{x \rightarrow 1-0} u_{0}(x)$. In this section, we consider the IBVP (1.1), (1.3), and (1.4) when

$$
u_{-}>u_{+}, \quad u_{-}^{1}>u_{+}^{1} \text {. }
$$


Let $v_{-}^{1}=\lim _{x \rightarrow 1-0} v_{0}(x)$ and $v_{+}^{1}$ be the solution to the following equation:

$$
\left(u_{-}^{1}-u_{+}^{1}\right)^{2}=\left(p\left(v_{-}^{1}\right)-p\left(v_{+}^{1}\right)\right)\left(v_{+}^{1}-v_{-}^{1}\right),
$$

with $v_{-}^{1}>v_{+}^{1}$.

Denote

$$
\begin{aligned}
& \beta_{0}=\left|v_{+}-v_{-}\right|+\left|u_{+}-u_{-}\right|+\left|v_{+}^{1}-v_{-}^{1}\right|+\left|u_{+}^{1}-u_{-}^{1}\right|, \\
& N_{5}=\max _{x \in(0,1)}\left\{\sum_{i=0}^{2}\left|\partial_{x}^{i}\left(v_{0}(x)-\bar{v}_{1}\right)\right|+\sum_{i=1}^{2}\left|\partial_{x}^{i} u_{0}(x)\right|\right\}, \\
& N_{6}=\max _{x \in(0,1)}\left\{\left|\partial_{x}^{3}\left(v_{0}(x)-\bar{v}_{1}\right)\right|+\left|\partial_{x}^{3} u_{0}(x)\right|\right\} .
\end{aligned}
$$

We have the following main result.

Theorem 3.1. Assume that $\beta_{0} \ll 1,\left(u_{0}, v_{0}\right) \in C^{3}((0,1))$ with $N_{5}+N_{6}<+\infty$, and $u_{i} \in C^{3} \cap H^{3}((0,+\infty))$. Then there exists an $\varepsilon_{3}>0$ such that if $N_{5}+\mu_{0}<\varepsilon_{3}$, the global weak entropy solution $(u, v)(x, t)$ to the IBVP $(1.1),(1.3),(1.4)$, and (3.1) exists, namely, two continuous and piecewise smooth curves $y_{i}=y_{i}(t): R_{+} \rightarrow[0,1]$ exist and satisfy $y_{1}(0)=1, y_{2}(0)=0, \dot{y}_{1}(t) \cdot \dot{y}_{2}(t)<0, \dot{y}_{i}(t)=\dot{x}_{1}(t)$ for $\dot{y}_{i}(t)<0$, and $\dot{y}_{i}(t)=\dot{x}_{2}(t)$ for $\dot{y}_{i}(t)>0$, where $x=x_{i}(t)(i=1,2)$ are denoted by (2.1) and $(2.2)$ respectively. Moreover, away from $x=y_{i}(t),(u, v)(x, t) \in C^{3}$, and, along $x=y_{i}(t)$, the Rankine-Hugoniot condition and the Lax entropy condition hold. In addition, as $t$ tends to infinity,

$$
\left|\partial_{x}^{i}\left((u, v)\left(y_{j}(t)+0, t\right)-(u, v)\left(y_{j}(t)-0, t\right)\right)\right| \sim O(1) e^{-\beta_{6} t} \rightarrow 0, \quad j=1,2, i=0,1,2,
$$

and

$$
\left\|\left(u, v-\bar{v}_{1}\right)(\cdot, t)\right\|_{2}^{2} \sim O(1)\left(e^{-\beta_{7} t}+A(t)\right) \rightarrow 0
$$

with two positive constants $\beta_{6}$ and $\beta_{7}$.

REMARK 3.2. The similar result to Theorem 2.4 is true in this case.

The proof of Theorem 3.1 is similar to that of Theorem 2.1, but it is more complicated since we also need to deal with the possible interactions of the shock discontinuities in different families and in the same family. For the interaction of shocks in different families, it can be done by solving locally a Riemann problem $[13,14]$ where one can obtain the similar estimates along shock waves and away from them. The shock interactions in the same family are more complicated because there will appear a rarefaction wave after the interaction. However, this case will never appear, because we can prove that if for some time $T>0$, there are two shock waves in the same family, the distance between them is uniformly bounded below if the assumptions of initial-boundary data in Theorem 3.1 are satisfied. Then, one of the shocks in the same family will hit the boundary and reflect to form a new shock wave in the other family. We omit the details here.

Acknowledgments. The second author thanks Professor Tao Luo for his kind help and suggestions. 


\section{REFERENCES}

[1] R. Courant and K. O. Friedrichs, Supersonic flow and shock waves, Interscience Publishers, Inc., New York, NY, 1948

[2] C. M. Dafermos, A system of hyperbolic conservation laws with frictional damping. Theoretical, experimental, and numerical contributions to the mechanics of fluids and solids, Z. Angew. Math. Phys. 46, Special Issue, S294-S307 (1995)

[3] L. Hsiao, Quasilinear hyperbolic systems and dissipative mechanisms, World Scientific, River Edge, NJ, 1998

[4] L. Hsiao and Hailiang Li, The system of compressible adiabatic flow through porous media with boundary effects, preprint, 1999

[5] L. Hsiao and T. P. Liu, Convergence to nonlinear diffusion waves for solutions of a system of hyperbolic conservation laws with damping, Comm. Math. Phys. 143, 599-605 (1992)

[6] L. Hsiao and T. P. Liu, Nonlinear diffusive phenomena of nonlinear hyperbolic systems, Chinese Ann. of Math. 14B, 465-480 (1993)

[7] L. Hsiao and T. Luo, Nonlinear diffusive phenomena of solutions for the system of compressible adiabatic flow through porous media, J. Differential Equations 125, 329-365 (1996)

[8] L. Hsiao and T. Luo, Nonlinear diffusive phenomena of entropy weak solutions for a system of quasilinear hyperbolic conservation laws with damping, Quart. Appl. Math. 56, 173-189 (1998)

[9] L. Hsiao and R. H. Pan, Damped P-system on bounded domain, Contemporary Mathematics 255, 109-124 (2000)

[10] L. Hsiao and R. H. Pan, Initial-boundary value problem for the system of compressible adiabatic flow through porous media, J. Differential Equations 159, 280-305 (1999)

[11] L. Hsiao and D. Serre, Global existence of solutions for the system of compressible adiabatic flow through porous media, SIAM J. Math. Anal. 27, 70-77 (1996)

[12] L. Hsiao and D. Serre, Large-time behavior of solutions for the system of compressible adiabatic flow through porous media, Chinese Ann. of Math. 16B, 431-444 (1995)

[13] L. Hsiao and S. Q. Tang, Construction and qualitative behavior of solutions for a system of nonlinear hyperbolic conservation laws with damping, Quart. Appl. Math. 53, 487-505 (1995)

[14] L. Hsiao and S. Q. Tang, Construction and qualitative behavior of the solution of the perturbated Riemann problem for the system of one-dimensional isentropic flow with damping, J. Differential Equations 123, 480-503 (1995)

[15] O. A. Ladyzhenskaya, The boundary value problems of mathematical physics, Applied Mathematical Sciences, Vol. 49, Springer-Verlag, New York, NY, 1985

[16] H. L. Li, The asymptotic behavior of solutions to the damped P-system with boundary effects, J. Partial Differential Equations 12, 357-368 (1999)

[17] T. T. Li and W. C. Yu, Boundary value problems for quasilinear hyperbolic systems, Duke Univ. Math. Ser. V, Durham, NC, 1985

[18] T. Luo and T. Yang, Global weak solutions for elastic equations with damping and different end states, Proc. Roy. Soc. Edinburgh Sect. A 128, 797-807 (1998)

[19] T. Luo and T. Yang, Interaction of elementary waves for compressible Euler equations with frictional damping, J. Differential Equations 161, 42-86 (2000)

[20] P. Marcati and M. Mei, Convergence to nonlinear diffusion waves for solutions of the initial boundary problem to the hyperbolic conservation laws with damping, Quart. Appl. Math. 58, 763-784 (2000)

[21] P. Marcati, A. Milani and P. Secchi, Singular convergence of weak solutions for a quasilinear nonhomogeneous hyperbolic system, Manuscripta Math. 60, 49-69 (1988)

[22] T. Nagasawa, Global asymptotics of the outer pressure problem with free boundary, Japan J. Appl. Math. 5, 205-224 (1988)

[23] K. Nishihara, Convergence rates to nonlinear diffusion waves for solutions of system of hyperbolic conservation laws with damping, J. Differential Equations 131, 171-188 (1996)

[24] K. Nishihara and T. Yang, Boundary effect on asymptotic behavior of solutions to the P-system with damping, J. Differential Equations 156, 439-458 (1999)

[25] D. Serre and L. Xiao, Asymptotic behavior of large weak entropy solutions of the damped p-system, J. Partial Differential Equations 10, 355-368 (1997)

[26] L. A. Ying and J. H. Wang, Global solutions of the Cauchy problem for a nonhomogeneous quasilinear hyperbolic system, Comm. Pure Appl. Math. 33, 579-597 (1980) 
[27] Y. S. Zheng, Global smooth solutions to the adiabatic gas dynamics system with dissipation terms, Proceeding of Sixth International Conference on Hyperbolic Problems, Theory, Numerical, Applications, Hong Kong, 1996 